\title{
Hemangioma mamario en una lactante con aparente mastitis. Caso clínico
}

\author{
Mammary hemangioma in an infant with apparent mastitis. Case report
}

\author{
Dra. Cristina Rodríguez Jiménez, ${ }^{a}$ Dra. Concepción Míguez Navarro, ${ }^{b}$ Dra. Minia Campos Domínguez, \\ Dra. Carmen Garrido Colino
}

\section{RESUMEN}

La patología mamaria en pediatría es infrecuente. El hallazgo de una masa a nivel mamario en un lactante es una situación poco común. Los posibles diagnósticos a esta edad son absceso mamario, mastitis, ingurgitación mamaria por estimulación hormonal materna y hemangioma. Es importante llegar al diagnóstico adecuado para emplear un tratamiento acorde y evitar la aparición de complicaciones de dichas patologías. Se presenta un caso de una lactante con una masa en la mama derecha detectada desde el nacimiento. Inicialmente, se trató como una mastitis, pero dada la mala evolución, se plantearon diagnósticos diferenciales y se concluyó que se trataba de un hemangioma. Debido a la ulceración de la lesión, junto con el riesgo existente de desarrollar hipoplasia mamaria, se decidió iniciar tratamiento con propanolol, con resolución casi completa de la tumoración.

Palabras clave: hemangioma infantil, enfermedades de la mama, úlcera, propanolol.

\begin{abstract}
Mammary pathology is infrequent during childhood. It is rare the probability of finding a breast mass in an infant. The most frequent possible diagnoses at this age are breast abscess, mastitis, breast engorgement due to maternal hormonal stimulation and hemangioma. Reaching the proper diagnosis is essential in order to apply a suitable treatment and avoid the potential disease complications. We present the case of a female infant having a mass in the right breast from birth. Initially the entity was treated as mastitis. Nevertheless, the bad evolution made necessary considering the differential diagnosis. It was concluded to be a hemangioma. Due to the lesion ulceration and the potential risk of developing breast hypoplasia, treatment with propranolol was started. The tumor was almost completely resolved.

Key words: infantile hemangioma, breastdiseases, ulcer, propanolol.
\end{abstract}

a. Pediatría, Hospital General Universitario Gregorio Marañón, Madrid, España.

b. Pediatría, Unidad de Urgencias Infantil, Hospital General Universitario Gregorio Marañón, Madrid, España.

c. Dermatología Infantil, Hospital General Universitario Gregorio Marañón, Madrid, España.

d. Pediatría, Servicio de Oncohematología Infantil, Hospital General Universitario Gregorio Marañón, Madrid, España.

Correspondencia:

Dra. Cristina Rodríguez Jiménez: cristinarj28@gmail.com

Financiamiento: Ninguno.

Conflicto de intereses: Ninguno que declarar.

Recibido: 8-3-2017

Aceptado: 26-7-2017 http:/ / dx.doi.org/10.5546/ aap.2018.e98

Cómo citar: Rodríguez Jiménez C, Míguez Navarro C, Campos Domínguez, et al. Hemangioma mamario en una lactante con aparente mastitis. Caso clínico. Arch Argent Pediatr 2018;116(1):e98-e101.

\section{INTRODUCCIÓN}

La patología mamaria en la edad pediátrica es poco frecuente. La aparición de una masa o tumoración localizada a nivel mamario puede deberse a diversas etiologías. En la mayoría de las ocasiones, suele tratarse de una causa benigna y autolimitada, pero aun así, puede generar una angustia familiar importante. A la hora de realizar un diagnóstico diferencial, hay que tener en cuenta la edad del paciente, pues según esta, será más probable una etiología u otra.

En los lactantes, los posibles diagnósticos de masas mamarias son absceso mamario, mastitis, ingurgitación mamaria por estimulación hormonal materna y hemangioma. Para llegar al diagnóstico definitivo, es importante indagar sobre el momento y el modo de aparición de la lesión, así como sobre su evolución, ya que el diagnóstico, en la mayoría de los casos, es clínico, sin necesidad de realizar pruebas complementarias. Solo en caso de que existan dudas diagnósticas, pueden llevarse a cabo pruebas: la ecografía es el estudio de imagen más útil para llegar al diagnóstico. Otra prueba que se puede emplear es la resonancia nuclear magnética (RNM), que permite evaluar la extensión en profundidad; sin embargo, no existe un consenso sobre cuándo se debe indicar. ${ }^{1}$

Una vez llegado al diagnóstico definitivo, se evaluará iniciar tratamiento, pues tan solo un $12 \%$ de los hemangiomas diagnosticados son los que lo recibirán. ${ }^{2}$ En la actualidad, el tratamiento de elección es el propanolol por vía oral; se deja a un lado el tratamiento corticoideo usado previamente. ${ }^{1,3}$ De esta manera, se evita la aparición de determinadas complicaciones; la más frecuente es la ulceración, que puede aparecer en un 5\%-10\% de los casos. ${ }^{4}$ No hay que olvidar otras 
complicaciones, como el compromiso de órganos vitales, que pueden surgir según la localización de los hemangiomas.

\section{Caso}

Lactante de sexo femenino de 60 días de vida, sin antecedentes de interés, derivado al Servicio de Urgencias hospitalario desde el centro de atención primaria por la sospecha de mastitis derecha de una semana de evolución que no mejoraba con ácido fusídico tópico y amoxicilina oral.

La madre refería aumento de volumen de la mama derecha desde el nacimiento y aparición de una lesión roja en el pezón de un mes de evolución que había ido aumentando. No refería la presencia de fiebre ni otra sintomatología asociada.

La exploración física mostraba la mama derecha con aumento de tamaño de consistencia blanda, sin aumento de la temperatura, con masa de $4 \times 4 \mathrm{~cm}$. En la areola y el pezón derecho, se observaba una lesión color rojo brillante de $1 \mathrm{~cm}$ de diámetro, de bordes bien definidos festoneados, ligeramente ulcerados y con descamación de piel (Figura 1.a y 1.b). Ante la palpación de la lesión, parecía no dolorosa.

Se hallaron dos lesiones papulares de color rojo vivo en la región inguinal derecha de 2-3 mm de diámetro (Figura 2).

En Urgencias, se solicitó una ecografía de la mama, y se observó una masa sólida de $2,8 \times 1,2 \mathrm{~cm}$ bien definida y polilobulada. El estudio con doppler mostró una masa muy vascularizada compatible con hemangioma infantil.

Con la sospecha diagnóstica de hemangioma infantil en la mama, fue derivada a consultas externas de Dermatología y Hematología Infantil, quienes, tras la evaluación, indicaron tratamiento con propanolol oral. Antes del inicio del tratamiento con beta bloqueantes, se realizó un estudio cardiológico con un electrocardiograma y una ecocardiografía sin alteraciones.

FIgura 1. a: Desigualdad en tamaño y coloración de ambas mamas. $\boldsymbol{b}$ : Incremento del tamaño de la mama derecha con lesión roja intensa en la areola y el pezón

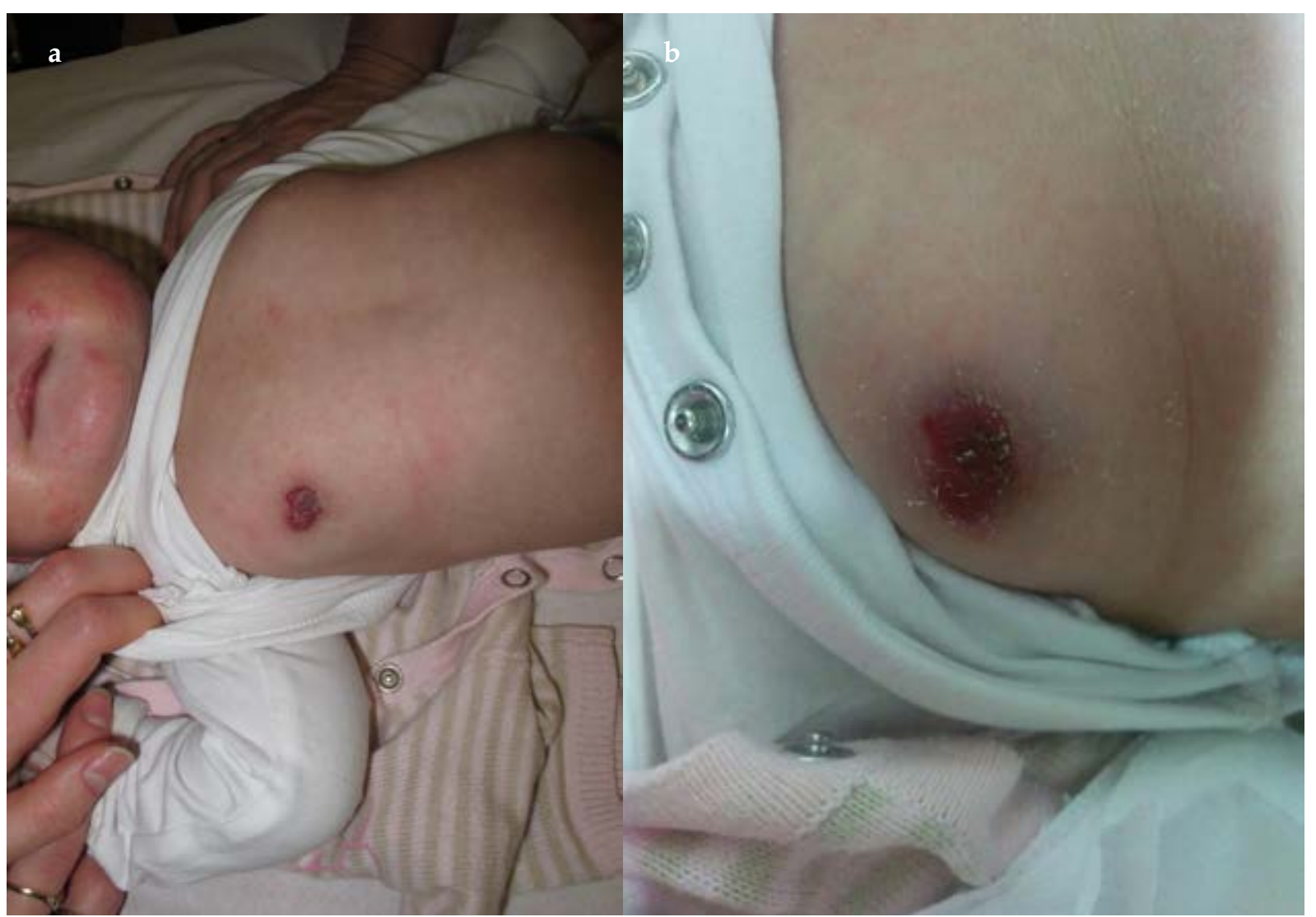


Posteriormente, se inició el tratamiento con propanolol en dosis bajas con aumento progresivo hasta $3 \mathrm{mg} / \mathrm{kg} /$ día, que se mantuvo durante 6 meses con adecuada tolerancia. Dada la buena evolución, se cambió el tratamiento a timolol tópico durante 2 meses más, con resolución de la lesión, y quedó únicamente una placa eritematosa residual de pequeño tamaño en el pezón derecho sin complicaciones añadidas.

\section{DISCUSIÓN}

El hemangioma infantil es el tumor vascular más frecuente en la infancia. Es más habitual en el sexo femenino en proporción 3:1 y en la raza blanca, como ocurre en nuestro caso. ${ }^{5}$ Se produce por proliferación de las células endoteliales de los vasos sanguíneos.

La historia natural es muy característica y se desarrolla en 3 etapas. La mayoría aparece a las 2-3 semanas de vida, y puede existir, en algunos casos, una lesión precursora desde el nacimiento, con crecimiento en los primeros meses de vida, que alcanza el $80 \%$ del tamaño final a los 4 meses. En esta etapa, es muy importante un seguimiento estrecho del paciente por la aparición de complicaciones, como se verá más adelante. Posteriormente, ocurre una fase en la que no se producen cambios en el hemangioma, conocida como fase de estabilización. Por último, una fase de involución espontánea que, generalmente, aparece tras el primer año de vida y suele tener una duración variable.
Se sitúa con más frecuencia ${ }^{6}$ en la cabeza y el cuello; sin embargo, puede encontrarse en cualquier localización, como, por ejemplo, en nuestro caso, en la mama.

Por la localización inusual, se debe plantear el diagnóstico diferencial con mastitis, absceso mamario e ingurgitación mamaria por estímulo hormonal. ${ }^{7}$ De hecho, la ingurgitación mamaria fisiológica que se produce por estímulo estrogénico es un factor predisponte para desarrollar una mastitis. A partir de las dos semanas de vida, la mastitis aparece con más frecuencia en el sexo femenino, con una proporción 2:1, debido a la mayor duración de la hipertrofia mamaria en las niñas. ${ }^{8}$

En nuestra paciente, la lesión mamaria se diagnosticó, inicialmente, como mastitis por la coloración rojiza y el aumento de tamaño de la mama. Sin embargo, no asociaba signos inflamatorios a nivel local ni lesiones en la piel de alrededor ni aumento del tamaño de los ganglios linfáticos axilares, que es característico de las mastitis. Tampoco presentaba síntomas sistémicos, como fiebre, irritabilidad y rechazo de tomas, como puede existir en los lactantes con mastitis. La importancia de llegar a un diagnóstico adecuado de mastitis se debe a que más de la mitad de los casos pueden progresar hacia la formación de un absceso mamario, ${ }^{9-11}$ así como la aparición de complicaciones, aunque estas son poco frecuentes. Las graves complicaciones que pueden producirse a partir

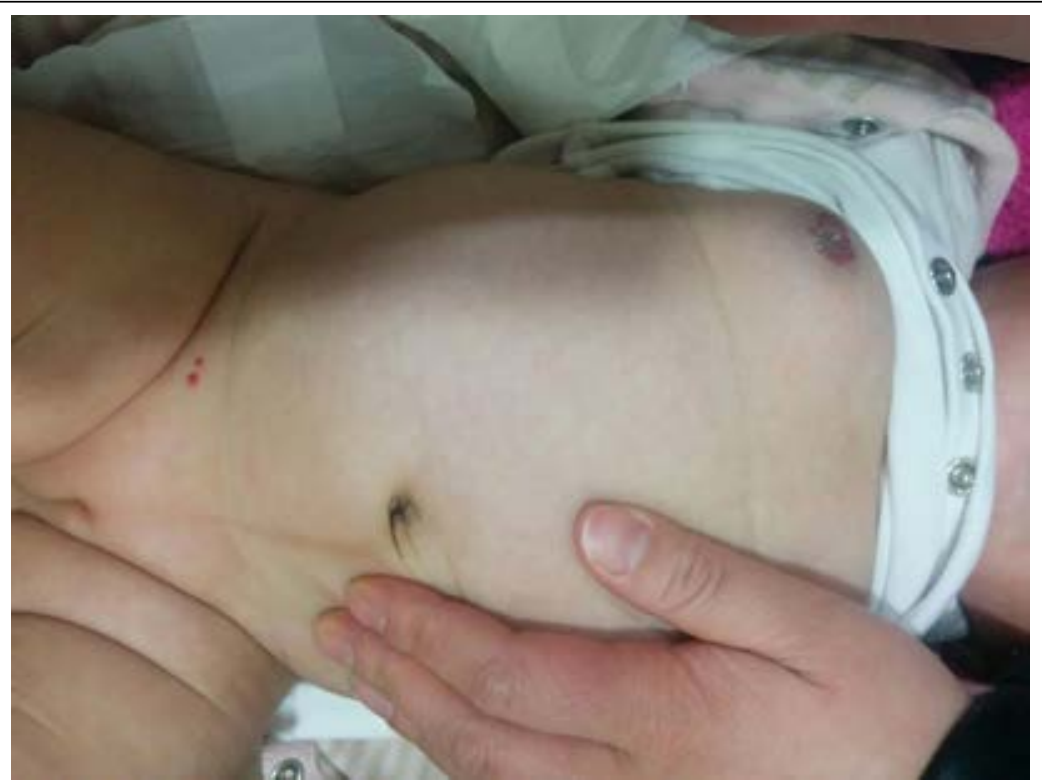


de una mastitis son celulitis extensa, fascitis necrotizante y osteomielitis. Para evitar estas consecuencias, se debe iniciar un tratamiento adecuado con antibioterapia, preferiblemente, por vía parenteral. El tratamiento por vía oral aumenta el riesgo de evolución de mastitis hacia absceso, que, además del tratamiento antibiótico, requerirá drenaje. ${ }^{12}$

En nuestra paciente, la combinación de una clínica poco concordante con mastitis y la ausencia de respuesta al tratamiento antibiótico obligó a pensar otro posible diagnóstico. Se llevó a cabo una prueba de imagen que ayudara a conocer la posible causa del crecimiento de dicha mama, que permitió llegar al diagnóstico definitivo de hemangioma.

Existen publicaciones que recomiendan realizar un estudio de extensión, con una ecografía abdominal, en busca de hemangiomas internos en caso de encontrar, durante la exploración física, 5 o más hemangiomas cutáneos. ${ }^{3}$ En nuestro caso, solo había tres lesiones compatibles con hemangiomas, por lo que este estudio no fue necesario.

La mayoría de los hemangiomas suelen tener un curso benigno, aunque en algunos casos, pueden sufrir complicaciones, sobre todo, a nivel local. La ulceración es la complicación más frecuente. ${ }^{1}$ Supone el 15,8\% de los casos y aparece, principalmente, durante la fase proliferativa y puede provocar sangrado y dolor. Existen varias localizaciones en las que la ulceración se produce con más frecuencia. Una de ellas es en las zonas de roce, como ocurre en nuestro caso. En la localización mamaria en niñas, también existe el riesgo de hipoplasia unilateral de la glándula y asimetría mamaria en el futuro. Entre las indicaciones actuales de tratamiento con propanolol, están la ulceración y la prevención de secuelas estéticas y funcionales, ${ }^{1}$ como fue nuestro caso. Aunque se ha confirmado la efectividad del tratamiento en las tres fases evolutivas del hemangioma, se ha demostrado que, durante la fase proliferativa, esta es mayor. Por lo tanto, se siguieron las recomendaciones actuales de iniciar el tratamiento de forma precoz en los primeros dos meses de vida.

\section{REFERENCIAS}

1. Baselga Torres E, Bernabéu Wittel J, van Esso Arbolave D, et al. Consenso Español sobre el hemangioma Infantil. An Pediatr (Barc) 2016;85(5):256-65.

2. Kilcline C, Frieden IL. Infantile hemangiomas: How common are they? A systematic review of the medical literature. Pediatr Dermatol 2008;25(2):168-73.

3. Abad ME, Angles V, Barabini LN, et al. Consenso sobre hemangiomas infantiles. Sociedad Argentina de Dermatología. 2016. [Consulta: 27 de julio de 2017]. Disponible en: http:/ / www.sad.org.ar/wp-content/ uploads / 2016 / 04 / HEMANGIOMAS-INFANTILESFINAL.pdf.

4. Chamlin SL, Haggstrom AN, Drolet BA, et al. Multicenter prospective study of ulcerated hemangiomas. J Pediatr 2007;151(6):684-9.

5. Haggstrom AN, Drolet BA, Baselga E, et al. Prospective study of infantile hemangiomas: demographic, prenatal, and perinatal characteristics. J Pediatr 2007;150(3):291-4.

6. Haggstrom AN, Drolet BA, Baselga E, et al. Prospective study of infantile hemangiomas: clinical characteristics predicting complications and treatment. Pediatrics 2006;118(3):882-7.

7. Greydanus DE, Matytsina L, Gains M. Breast disorders in children and adolescents. Prim Care 2006;33(2):455-502.

8. Walsh M, McIntosh K. Neonatal mastitis. Clin Pediatr (Phila) 1986;25(8):395-9.

9. Efrat M, Mogilner JG, Iujtman M, et al. Neonatal mastitis-diagnosis and treatment. Isr J Med Sci 1995;31(9):558-60.

10. Montague EC, Hilinski J, Andresen D, et al. Evaluation and treatment of mastitis in infants. Pediatr Infect Dis J 2013;32(11):1295-6.

11. Al Ruwaili N,' Scolnik D. Neonatal mastitis: controversies in management. J Clin Neonatol 2012;1(4):207-10.

12. De Silva NK, Fortunov R. Mastitis and breast abscess in infants younger than two months. UpToDate. [Consulta: 27 de julio de 2017]. Disponible en: https: / / www.uptodate. $\mathrm{com} /$ contents / mastitis-and-breast-abscess-in-infantsyounger-than-two-months. 